\title{
Research Paper: Translation, Validity, and Reliability of the Upper Extremity Fugl-Meyer Assessment (FMA-UE) in Persian Speaking Stroke Patients
}

\author{
Zahra Hassani $^{1}$, Hamid Reza Mokhtarinia ${ }^{2,3^{*}}$ (D), Amir Hossein Kahlaee ${ }^{3}$, Charles Philip Gabel ${ }^{4}$ (i)
}

1. Department of Occupational Therapy, Faculty of Rehabilitation, Semnan University of Medical Sciences, Semnan, Iran

2. Department of Ergonomics, Faculty of Rehabilitation, University of Social Welfare and Rehabilitation Sciences, Tehran, Iran.

3. Department of Physiotherapy, Faculty of Rehabilitation, University of Social Welfare and Rehabilitation Sciences, Tehran, Iran.

4. Physiotherapist, Coolum Beach, Sunshine Coast, QLD, Australia.

\begin{tabular}{|c|c|}
\hline $\begin{array}{l}\text { Use your device to scan } \\
\text { and read the article online }\end{array}$ & Chtat on Hassani Z, Mokhtarinia HR, Kahlaee AH, Gabel CP. Translation, Validity, and Reliability of the Upper Extremity \\
\hline 口ifisis & $\begin{array}{l}\text { Fugl-Meyer Assessment (FMA-UE) in Persian Speaking Stroke Patients. Iranian Rehabilitation Journal. 2022; 20(Special Is- } \\
\text { sue):37-46. http://dx.doi.org/10.32598/irj.20.Speciallssue.919.3 }\end{array}$ \\
\hline atsing & doi'http://dx.doi.org/10.32598/irj.20.SpecialIssue.919.3 \\
\hline
\end{tabular}

Article info:

Received: 28 Aug 2021

Accepted: 08 Oct 2021

Available Online: 01 Jan 2022

Keywords:

Persian, Fugl-Meyer Assessment, Measurment, Upper Extremity, Reliability, Stroke

\section{ABSTRACT}

Objectives: Fugl-Meyer Assessment (FMA) is the common scale for clinical and functional evaluation of sensorimotor conditions and related Upper Extremity (UE) dysfunction after stroke. This study was done to translate and cross-culturally adjust the original upper extremity FMA (FMA-UE) into Persian and to evaluate the psychometric properties of the translated version.

Methods: A procedure of forward/backward translation based on the published guidelines was adopted and two independent bilingual translators performed the translations in each stage. The conceptual and semantic equivalence was obtained through a consensus between experts. Consecutive stroke patients $(n=47$, male $=63 \%)$ with a mean age of $61.54 \pm 10.9$ years were recruited. Content, face, and concurrent validity was calculated using the content validity index, a cognitive interview, and correlation with the Wolf Motor Function Test (WMFT) Internal consistency and intra-rater reliability were determined by calculating Cronbach's alpha and the Intra-Class Correlation coefficient $\left(\mathrm{ICC}_{2.1}\right)$.

Results: During the forward translation and cultural adjustment, some wording changes were performed. In the forward translation, the most challenging clarifications are related to anatomical terms and positions. The total FMA-Persian score demonstrated acceptable internal consistency $(\alpha=0.86)$ and intra-rater reliability $\left(\mathrm{ICC}_{21}=0.96\right)$. Joint passive motion showed the lowest reliability among all domains. The FMA motor subscales showed a floor effect, while sensation, joint passive motion, and pain domains showed ceiling effects. The correlation between the FMA-UE score and the WMFT was $0.78(\mathrm{P}<0.001)$.

Discussion: The FMA-UE translation and adjustment were performed successfully into the Persian language. The results of the current study found FMA-UE as an acceptable, reliable, and valid instrument for evaluating the upper limb function after stroke in Persian-speaking patients. However, it should be noted that floor and ceiling effects are respectively present in the domains of the motor subscales and for sensation, passive motion, and pain.

\section{* Corresponding Author:}

Hamid Reza Mokhtarinia, PhD.

Address: Department of Ergonomics, Faculty of Rehabilitation, University of Social Welfare and Rehabilitation Sciences, Tehran, Iran.

Tel: +98 (21) 22180119

E-mail: hrmokhtarinia@yahoo.com 


\section{Highlights}

- Disability of the upper limb after stroke is a major concern adversely affecting the quality of life in these patients.

- Stroke rehabilitation requires a reliable and valid tool to assess sensorimotor functioning of the upper limb.

- Fugl-Meyer Assessment of the Upper Extremity (FMA-UE) is one of the most frequently used instruments to quantitatively assess post-stroke upper limb disability and recovery.

- Translation, cultural adjustment of the original version, and psychometric evaluation of the Persian version of FMAUE (FMA-UE-Pr) were presented in this work.

- The Persian version of the FMA-UE was developed from the original and was found as a reliable and valid instrument for the evaluation of clinical and intervention effects.

\section{Plain Language Summary}

Function and mobility limitations after stroke are the two most common dysfunctions that may interfere with stroke patients' activities of daily living. To monitor sensorimotor improvements following the rehabilitation intervention for patients, a valid, reliable, practical, and efficient instrument is required. Among the available instruments, the FMA-UE is the most valid and reliable instrument and has been translated into various languages; however, there is no Persian version. To compare the treatment results of different counties and because of possible cultural differences, a psychometric evaluation of the Persian version of the FMA-UE is needed. The present study reported the psychometrics evaluation results of the FMA for upper limb disorders after stroke.

\section{Introduction}

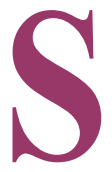

troke is one of the major global health problems with high mortality (5.5 million) and high related disability in survivors $(50 \%)$ [1]. The prevalence of stroke has an increasing worldwide trend. The American Heart Association working group has estimated that by 2030 , around $4 \%$ of US adults will have had a stroke. In concurrence with this, East Asia has one of the highest incidence rates of stroke followed by the regions of Eastern Europe and Latin America [2]. In Iranian people, the situation is comparable to other countries with the incidence of stroke reported at around 43 patients per 100,000 population [3] .

Residual deficits and disability in stroke survivors will remain for several years after stroke [4]. Sensory and motor deficits in the upper extremity are the two most common impairments associated with stroke, which creates functional and mobility limitations along with problems in the areas of balance, handling, writing, and normal daily function $[4,5]$. Evaluation of related impairments post-stroke is essential to plan for effective rehabilitation programs, understanding the motor control mechanisms and motor learning processes [6]. When the magnitude and severity of impairment are evaluated accurately, a better prognosis and rehabilitation approach can be conducted.

There are various instruments and scales to evaluate upper extremity sensory-motor function in hemiparetic patients. A validated instrument is necessary to monitor any motor and sensory improvement following the rehabilitation intervention of stroke patients [7]. Further, any such instrument should be practical, less consuming, efficient reliable, and demonstrate high utility in both the research and clinical settings [8].

The Fugl-Meyer Assessment of the Upper Extremity (FMA-UE) is a quantitative instrument originally developed in English by Fugl-Meyer et al. in 1975 [9]. Results of the reliability, validity, and feasibility of the FMA demonstrate that it is a high-quality instrument, which is used for the evaluation of the upper extremity in stroke patients [10]. Consequently, the FMA-UE is considered a suitable clinical outcome in motor, sensory, and functional evaluation of neurological conditions involving the upper extremity $[11,12]$. Psychometric evaluation of a culture-matched instrument should be performed specifically in each individual language and culture. Various translated and adapted versions of the 
FMA-UL have been provided in various English speaking [13-15] and non-English speaking countries [16, 17]. Despite the fact that there are more than 110 million Persian-speaking people worldwide (mostly resident in the Middle East), FMA-UE has not yet been prepared for use in this large population. A Persian version of the FMA-UL can help in the sensorimotor assessment procedures in Persian-speaking patients with stroke and obtain results that will be comparable with results from other countries. Therefore, the aim of the current study was to translate and cross-culturally adjust the FMA-UL to Persian and confirm its clinometric properties, including content validity, reliability, and internal consistency in stroke patients.

\section{Materials and Methods}

\section{Fugl-Meyer Assessment-Upper Extremity (FMA-UE)}

The FMA-UE is a condition-specific outcome that applies to hemiparesis of the upper limb in stroke conditions. The FMA-UE consists of three domains: motor, sensory and passive joint motion/pain. The motor domain includes 33 items (4-subtests) with scores ranging 0-66 points; the sensation section includes 6 items (2-subtest) with a score range of 0-12 points; with the passive joint domain including 24 items (2-subtest) with a score range of $0-48$ points. All items are scored ordinal on a 3-point scale. A total score is calculated by summing all the calculated item scores with a range of $0-126$ points $[7,18]$.

\section{Wolf Motor Function Test (WMFT)}

WMFT is an observational functional tool, including 15 functional tasks, which are observed and scored by the therapist and is used commonly for the assessment of upper extremity function in stroke patients. Performances were rated using a functional ability scale with six points. The Persian version of the WMFT showed acceptable reliability and validity (ICC range, 0.97-0.99) [19].

\section{Translation and cross-cultural adjustment}

The FMA-UE includes two sections, a scoring sheet, and a related manual. In this study, both sections were considered for translation into Persian according to published procedures $[20,21]$ and suggested guidelines [22]. Firstly, permission for the Fugl-Meyer Assessment protocols was obtained from Dr. Margit Alt Murphy at the University of Gothenburg. The original version was translated from English to the Persian language by two native Persian speakers, a physiotherapist, and a profes- sional translator. The two translators and two research team members discussed any discrepancies and a final consensus agreement was achieved. Backward translation was performed by two further independent bilingual and blinded translators. In an expert panel composed of one occupational therapist, one neurologist, one physiotherapist, one psychologist, and the translators, the original and backward translation versions were compared. Following the resolution of any discrepancies, a pre-final version was produced.

This version was sent to Dr. Margit to clarify any semantic, idiomatic, and conceptual equivalence with the original version. After several rounds, a final confirmed equivalency was reached.

\section{Participants}

All participants were recruited from a sample of convenience at the Rofideh Rehabilitation and Noor Afshar Hospital in Tehran from Nov 2019 to Feb 2021. A total of 47 patients (29 male, 18 female, age $40-80$ years) suffering from ischemic or hemorrhagic stroke were recruited. All the participants were diagnosed and referred by a neurologist as having incomplete upper extremity paresis related to their stroke and were able to personally provide written informed consent.

The exclusion criteria were obvious dementia, any mental deficit, which may affect cognitive performance, function limitation as a result of moderate to severe pain, and inability to follow the verbal instructions by the assessor [7]. The ethics approval for this study was provided by the committee of the University of Social Welfare and Rehabilitation Sciences (USWR) (IR.USWR. REC.1398.151).

\section{Psychometric evaluation}

Face validity: This was determined through the translation process involving the suggested alternative wording, checking the understandability, readability, and cultural relevance of the translation [23]. Face validity was evaluated on a sample of eight physiotherapists (four) and occupational therapists (four) through a 15-minute cognitive interview.

Content validity: The same expert panel used in the face validity process evaluated the content validity using a 4-point Likert scale for each item relevance to the construct of upper extremity functional deficit $(1=$ not relevant, $2=$ somewhat relevant, $3=$ quite relevant, and 4=highly relevant). Subsequently, the Content Validity 
Index (CVI) was determined at two levels of the item (ICVIs) and scale (S-CVI) [24, 25]. The accepted values for the I-CVI and S-CVI values are considered above 0.78 and 0.90 , respectively, which represents evidence of high content validity value for the scale [24].

Concurrent validity: Concurrent validity measures how well a new test compares to a valid established test. WMFT is a valid test in Persian for assessing the function of stroke patients. This assessment was done by determining the correlation between the MAS-UE total score and domains with the WMFT score.

Reliability: A sub-sample of 30 patients participated to assess the reliability. Two aspects of reliability were evaluated: relative and absolute reliability. Relative reliability was determined for intra-rater reliability of both the total score and subscores using the interclass correlation coefficient type 2,1 $\left(\mathrm{ICC}_{2,1}\right)$ and the related two-sided 95\% Confidence Interval (CI). A random subgroup $(n=20)$ of the full 47 entered patients was taken. The $\mathrm{ICC}_{2,1}$ value of 0.8 was considered 'good' and $>0.9$ as excellent [26, 27]. Cronbach's alpha was used as a measure of internal consistency and calculated where a value $>0.70$ was considered acceptable $[28,29]$. To test the absolute reliability, the Standard Error of Measurement (SEM) was chosen. SEM was calculated using the following formula: SEM=SD, where SD and ICC are the measurement standard deviation and correlation between two measurements, respectively.

Floor/ceiling effects: The floor and ceiling effects related to the scoring distribution were evaluated. The floor effect is defined as the percentage of the individuals in a sample with the worst possible scores, and the ceiling effect indicates cases with the best scores. If $>15 \%$ of the sample achieved these minimum or maximum scores, then, floor and/or ceiling effects are present [20, 30].

\section{Statistical analysis}

Participants' characteristics and the score distributions of the scales and subscales were analyzed and reported as frequencies, percentages, means, and Standard Deviations (SDs). To determine the intra-rater reliability, the $\mathrm{ICC}_{2,1}$ and $95 \% \mathrm{CI}$ were calculated. SEM was calculated from the obtained data of the relative reliability and SD of two trial measurements. The concurrent validity was measured by calculating Spearman's correlation coefficient between FMA-UE and MAS-UE scores. Correlations greater than 0.75 were considered good to excellent [16]. Data analysis was performed using the statistical package for social science v. 16 SPSS software for windows and all statistical significance was considered at $\mathrm{P}<0.05$.

\section{Results}

\section{Cross-cultural adjustment process}

In the forward translation, the most challenging clarifications were related to anatomical terms and positions, which have no Persian equivalent. Hence, we faced difficulty in terms of whether they should be translated or the same words used but in the Persian writing form. Further, some terms have existing Persian equivalents and translations, but due to the frequent use of the English word itself, it is often easier for professionals who must complete the questionnaire to use the original word. Conversely, considering the need to conduct psychometric evaluation, translation and cultural adjustment should be considered exactly. For example, the "بازتاب" (//baaztaab//) is the translation of "reflex" in Persian, but in examining face validity, many experts did not agree with this term as it was thought the word was unfamiliar. Finally, it was decided to write the Persian equivalent of such words and include the English format in Persian in parentheses. Other words in the text included were as follows: "Flexor, extensor, retraction, supination, synergy, opposition, grasping, and dysmetria".

All of these terms were approved and the consensus was obtained by the expert panel in a meeting with the presence of translators and researchers of the project and the aforementioned physiotherapists and occupational therapists. In order to maintain the intention of the original concept and the scale, the final approval by the original author was also obtained.

In the backward translation process, due to the existence of a Persian equivalent for several English words, we encountered some translation problems. For example, "anesthesia" and "numbness" are translated as a single word in Persian. Consequently, in the " $H$ " section of the questionnaire, disagreement was present on whether the translation should be numbness or anesthesia. In the "C" section, the translation of the word "interposed" was difficult because there was no Persian equivalent. First, it was translated to "between hands" but for more clarification and after discussion with the original developer and the research team members the phrase "objects near the palm of the hand" was chosen.

In section 5 (normal reflex activity), the reflex activity was classified into three categories: hyper, lively, and normal. There was no equivalent for the word lively in Persian, and 
Table 1. Demographic data on the participants and the mean Fugl-Meyer Scale scores

\begin{tabular}{cc}
\hline Demographic Data & Mean \pm SD or No.(\%) \\
\hline Age (y) & $61.54 \pm 10.9$ \\
\hline Sex (Female/Male) & $29(63) / 17(37)$ \\
\hline Infarct/Hemorrhage & $31(67.3) / 15(32.7)$ \\
\hline Side of paresis (Right/Left) & $27(58.7) / 19(41.3)$ \\
\hline Time since the onset of stroke (month) & $5.7 \pm 1.2$ \\
\hline Total FMA & $73.78 \pm 22.57$ \\
\hline Motor section & $19.22 \pm 3.56$ \\
\hline A. Shoulder/elbow/forearm & $12.84 \pm 5.32$ \\
\hline B. Wrist & $2.95 \pm 1.8$ \\
\hline C. Hand & $4.68 \pm 2.91$ \\
\hline D. Coordination/speed & $2.92 \pm 1.24$ \\
\hline H. Sensation section & $10.52 \pm 2.33$ \\
\hline I. Joint passive motion & $23.28 \pm 1.29$ \\
\hline J. Pain section & $20.76 \pm 4.49$ \\
\hline
\end{tabular}

Iranian Rehabilitation Journa

Table 2. Agreement percentages of experts on the face validity of FMA-UE

\begin{tabular}{cccc}
\hline & & $\%$ & \\
\hline Fugel-Meyer Assessment Items & Necessity & Clarity & Simplicity \\
\cline { 2 - 4 } & 100 & 100 & 85 \\
\hline A1 & $85-100$ & $71-85$ & 85 \\
\hline A3 (A3.1- A3.9) & $85-100$ & $66-100$ & 85 \\
\hline A4 (A4.1- A4.9) & $85-100$ & $85-100$ & 100 \\
\hline A5 (A5.1- A5.3) & $85-100$ & $85-100$ & 85 \\
\hline B (B1-B15) & $71-85$ & $83-100$ & 83 \\
C (C1-C17) & 100 & 100 & 85 \\
D & 100 & 85 & 100 \\
H & 100 & 100 & 100 \\
\hline I & 100 & 100 & 100 \\
\hline J & 100 & 100 & 100 \\
\hline
\end{tabular}


Table 3. Intra-Class Correlation coefficient (ICC) values for FMA-UE sections

\begin{tabular}{ccccc}
\hline \multirow{2}{*}{ Fugl-Meyer Assessment } & \multicolumn{3}{c}{ Intraclass Correlation Coefficient } & \multirow{2}{*}{ SEM } \\
\cline { 2 - 4 } Total FMA & ICC & $95 \% \mathrm{CI}$ & P-Value & 4.73 \\
Motor section & 0.96 & $0.92-0.99$ & 0.0001 & 3.54 \\
\hline A. Shoulder/elbow/forearm & 0.94 & $0.98-0.99$ & 0.0001 & 1.89 \\
B. Wrist & 0.96 & $0.98-0.99$ & 0.0001 & 1.25 \\
C. Hand & 0.97 & $0.92-0.99$ & 0.0001 & 1.34 \\
\hline D. Coordination/speed & 0.98 & $0.97-0.99$ & 0.0001 & 0.97 \\
\hline Sensation section & 0.98 & $0.95-0.99$ & 0.0001 & 1.04 \\
\hline Joint passive motion & 0.98 & $0.94-0.99$ & 0.0001 & 4.6 \\
\hline pain section & 0.88 & $0.63-0.96$ & 0.0001 & 5.1 \\
\hline
\end{tabular}

Пranian Rehabilitation \ournal

after discussing with the original developer "exaggerated" indicating increased reflex activity was approved.

In section $\mathrm{D}$, in order to better understand the term "Dysmetria" and its grading, some explanations (irregular distance from the tip of the nose in movement repetitions) were added in front of "pronounced or unsystematic". Also, for more clarification of "slight and systematic" the "distance from the tip of the nose is small and regular" was added in front.

In the final version, the title of "FMA-UE protocol Persian version" and "Rehabilitation Medicine, University of Gothenburg" was added in the header of the questionnaire. The above corrections were made in agreement with Dr. Margaret, who approved the final version after several rounds of sharing the questionnaire (Appendix 1).
Participants

Table 1 depicts the participants' $(n=47)$ demographic characteristics. Male participants were higher in number $(n=29,63 \%$, age $=61.79 \pm 9.66$ years $)$ compared to the females $(n=17,37 \%$, age $=61.12 \pm 13.07$ years $)$.

Evaluation of the psychometric properties

\section{Face validity}

Face validity of the FMA-UE-Pr translation was evaluated by eight experts through a committee discussion and the pilot test. They agreed on the clarity, simplicity, and necessity of the questionnaire's items to assess the upper extremity functional status post-stroke. The results of agreement on each of the items and scales are reported in Table 2.

Table 4. Concurrent validity by spearman's correlation analyses

\begin{tabular}{cccc}
\hline \multirow{2}{*}{ FMA-UE Score } & \multicolumn{3}{c}{ WMFT Score } \\
\cline { 2 - 4 } & R Spearman & P-Value & 95\% Cl \\
\hline Total (0-126) & 0.78 & 0.004 & $0.68-0.91$ \\
\hline Motor section (0-66) & 0.74 & 0.003 & $0.68-0.84$ \\
\hline Sensation section (0-12) & 0.18 & 0.21 & $-0.11-0.34$ \\
\hline Joint motion/pain 90-48) & 0.21 & 0.26 & $0.05-0.31$ \\
\hline
\end{tabular}

Iranian Rehabilitation Journal 
Table 5. Floor and ceiling effects

\begin{tabular}{|ccc}
\hline \multirow{2}{*}{ Fugl-Meyer Assessment } & \multicolumn{2}{c}{ Ceiling and Flooring Effects Results } \\
\cline { 2 - 3 } & Floor, \% & \%Ceiling, \% \\
\hline Total FMA (0-126) & 0 & 2.2 \\
\hline Motor section (0-66) & 4.3 & 0 \\
\hline A.Shoulder/elbow/forearm (0-36) & 8.7 & 0 \\
\hline B. Wrist (0-10) & 45.7 & 8.7 \\
\hline C. Hand (0-14) & 43.5 & 10.9 \\
\hline D. Coordination/speed (0-6) & 45.7 & 8.7 \\
\hline H.Sensation section (0-12) & 0 & 65.2 \\
\hline I. Joint passive motion (0-24) & 6.5 & 73.9 \\
\hline J. pain section (0-24) & 2.2 & 30.4 \\
\hline
\end{tabular}

Iranian Rehabilitation Journal

\section{Content validity}

The results of the CVI showed suitable content validity for both items and scales. The isolated relevance of items $\mathrm{A} 1, \mathrm{~A} 2, \mathrm{~A} 3, \mathrm{~A} 4$, and A5 was $100,71,85,100,85 \%$, respectively. The related values for sections $\mathrm{B}, \mathrm{C}$, and $\mathrm{D}$ were $85 \%$ and for $\mathrm{H}$, $\mathrm{I}$, and $\mathrm{J}$ they were $100 \%$. Further, in order to check the total content validity (S-CVI), the average values were calculated with the S-CVI for section $\mathrm{A}$ is $88.2 \%$ and for the whole questionnaire is $91.8 \%$.

\section{Reliability}

The reliability results showed excellent intra-rater reliability for the total score and all sections, except the joint passive motion and pain sections, which showed 'good' reliability. The $\mathrm{ICC}_{2.1}$ value results regarding the relative reliability and SEM value related absolute reliability are presented in Table 3. Further, the internal consistency for the motor section and the total score was 0.88 and 0.86 , respectively.

\section{Concurrent validity}

The results showed a positive significant correlation between the FMA-UE total score, motor domain and the WMFT with a Spearman's correlation coefficient of 0.78 and 0.74 , respectively. A week correlation between the sensation, joint motion/pain domain score and WMFT score confirmed the discriminant validity of the FMA-UE for sensation domain and also the joint motion/pain domain (Table 4).

\section{Ceiling and flooring effects}

The FMA-UE total score and motor domain did not show floor or ceiling effect. However, the subscale for motor showed a floor effect, and the subscale for sensation, joint passive motion, and pain domain showed ceiling effects effect (Table 5).

\section{Discussion}

The current study is the first to report psychometric evaluation of the FMA-UE in Persian-speaking Iranian stroke patients. Translation and cultural adjustment of a scale, which is developed in an original language, is required to be used in different languages in clinical and scientific settings. This study was conducted according to the previous published guidelines of translation and cultural adjustment procedures [22] to reach a maximum semantic-conceptual equivalence between the Persian and the original version of FMA-UE.

The original FMA-UE scale is freely available at www. neurophys.gu.se/rehabmed, and is validated in English, Italian [31], Danish [16, 32], Korean [33], and Spanish [6]. This study provided the same equivalence in the Persian language. The cultural adjustment and translation results of the FMA-UE have been reported briefly in previous research in Brazilian [17], Danish [16], and Italian versions [31]. We encountered some challenges during the translation and adjustment and several items were changed as a direct consequence of cultural and linguistic differences. There was no equivalent for some an- 
atomical terms and positions in Persian. Consequently, translation was not required and the terms were written in the Persian script format. Some overlap is visible with these terms in translation to other language versions such as the Italian [31], and Spanish versions [6].

In backward translation, because of Persian similarity in the translation of some terms, such as anesthesia and numbness, which both have the same meaning of "بى "حسى (//bi-hessi//) in Persian, there was a challenge. In the "C" section, the phrase "interposed" and in the D section the "Dysmetria" were not familiar terms in Persian; thus, the consensus statement was to add some explanation for clarification. Interposed refers to not wanting the subject to perform an active grasp of the object. Its original meaning is for assessing the ability of the patients to open their fingers and hands to the size of the object and then demonstrate the ability to manipulate and hold it. Such descriptive expressions as used in our study were suggested in the Italian [31] and Spanish [6] versions.

In our study, reliability was evaluated on all the FMAUE domains in the stroke patients for the affected upper extremity. Most previous published papers only performed reliability analysis on a limited number of domains. Our results indicated that the FMA-UE has high reliability for all domains and the total score, which were similar to the results of previous research. Page et al. reported the ICC for the wrist and hand section of the FMA-UE for stroke patients in the US at 0.95 and 0.99 , respectively [34]. Roman et al. reported the ICC for a total score of 0.98 in a group of 64 post-stroke Romanian patients [35]. In the Japanese version of the FMAUE, ICC values were from 0.90-0.99 [7] for all domains. Similar results were reported by Sullivan et al. [12] and Lundquist et al. [16]. In the Danish version, Lin et al. showed good reliability on the sensory section of the FMA-UE (ICC=0.93) [36]. Platz et al. [18] reported an ICC range of 0.97-0.99 for all domains of FMA-UE in a European sample. The high reliability supports the use of the instrument for temporal follow-up as is required for determining the interventional effects.

The value for a 'good' internal consistency is reported in the range of $0.70-0.95$. Therefore, the Persian FMAUE (Alpha $=0.86$ ) has demonstrated this and is comparable with previously published versions, including the Danish version where Lin et al. showed 'excellent' internal consistency on the sensory section [36].

Impairment changes following the stroke can be evaluated by clinical measures, such as the Box-and-Block Test (BBT) [18], Motor Activity Log (MAL) [7], Ac- tion Research Arm Test (ARAT) [7], and the WMFT. In this study, due to the existence of the Persian version of WMFT, concurrent validity was evaluated through this instrument. WMFT is considered a gold measure for assessing the upper extremity function [19].

There was a significant correlation between the WMFT and both the total score of FMA-UE and the motor section, which indicates a good concurrent validity. The rest domains of the FMA-UE, including sensation and joint motion/pain domains showed a weak correlation with WMFT, which supports the discriminant validity of the FMA-UE. Amanoa et al. reported concurrent validity of FMA-UE with three instruments, ARAT, BBT, and MAL. Similar results with the current study were obtained with a spearman correlation coefficient of $0.94, .0 .94$, and 0.92 for ARAT, BBT, and MAL, respectively [7].

Roman et al. used the Functional Independence Measure (FIM) and the Modified Rankin Scale (MRS) to evaluate the concurrent validity and reported the correlation with FIM and MRS with a Pearson's correlation coefficient of 0.789 and -0.787 [35]. In the Danish version of FMA-UE, concurrent validity was performed using the Motor Assessment Scale and high correlation $(\mathrm{r}=0.94-0.95)$ was reported [16].

Floor and ceiling effects are only reported in a limited amount of previous research. In the Danish version assessed by Lin et al., the sensory section of the FMA-UE showed significant ceiling effects [36]. The possible causes can be inappropriate scoring steps, not considering other sensory evaluation, such as temperature, sense of effort, stereognosis, and alterations in two-point discrimination. With a high ceiling effect, the discriminative ability of an instrument is under question. Lundquist et al. showed no ceiling/floor effects at baseline or follow-up for the Danish version [16].

\section{Conclusion}

The Persian version of the FMA-EU was successfully achieved based on standard translation and cultural adjustment procedures. Generally, all domains showed 'good' reliability in assessing the affected upper extremities in stroke patients. Consequently, the Persian version of the FMA-UE is now translated as a valid and reliable scale for assessing upper limb function post-stroke.

\section{Strengths and limitations}

The major strength of this study is that it provides the FMA-UE as a valid and reliable questionnaire in the 
Persian language. Further, the reliability was tested on all domains and found to be comparable to the finding in other cultural and linguistic adjustments where such psychometric characteristics were considered. The limitations are related to the present sample data size being relatively small. A larger sample may be required to be examined to verify whether the results can be replicated. Also, the study was conducted on a sample of 'sub-acute and chronic stages of stroke patients, which may limit the generalizability of the results to acute samples till a separate specific sample is examined.

\section{Ethical Considerations}

\section{Compliance with ethical guidelines}

This study was approved by the Ethics Committee of the University of Social Welfare and Rehabilitation Sciences (IR.USWR.REC.1398.151). Informed consent was obtained from all participants included in the study.

\section{Funding}

This study was financially supported by the Clinical Research Development Center of Rofeideh Rehabilitation Hospital, USWR (Grant No: 2288)

\section{Authors' contributions}

Conceptualization and Supervision: Hamid Reza Mokhtarinia and Charles Philip Gabel; Methodology: Amir Hossein Kahlaee and Hamid Reza Mokhtarinia; Investigation, Writing - original draft, and Writing - review \& editing: All authors; Data collection: Zahra Hassani and Data analysis: Hamid Reza Mokhtarinia and Charles Philip Gabel; Funding acquisition and Resources: Amir Hossein Kahlaee and Hamid Reza Mokhtarinia.

\section{Conflict of interest}

The authors declared no conflict of interest.

\section{Acknowledgments}

The authors would like to acknowledge the kind assistance of the patients who contributed to the study. We also appreciate assistance received from the Clinical Research Development Center of Rofeideh Rehabilitation Hospital.

\section{References}

[1] Donkor ES. Stroke in the 21st Century: A snapshot of the burden, epidemiology, and quality of life. Stroke Research and Treatment. 2018; 2018:3238165. [DOI:10.1155/2018/3238165] [PMID] [PMCID]

[2] Gorelick PB. The global burden of stroke: Persistent and disabling. The Lancet Neurology. 2019; 18(5):417-8. [DOI:10.1016/ S1474-4422(19)30030-4]

[3] Daneshfard B, Izadi S, Shariat A, Toudaji MA, Beyzavi Z, Niknam L. Epidemiology of stroke in Shiraz, Iran. Iranian Journal of Neurology. 2015; 14(3):158-63. [PMID]

[4] Benjamin EJ, Blaha MJ, Chiuve SE, Cushman M, Das SR, Deo $\mathrm{R}$, et al. Heart disease and stroke statistics-2017 update: A report from the American Heart Association. Circulation. 2017; 135(10):e146-603. [PMID]

[5] Kim H, Her J, Ko J, Park DS, Woo JH, You Y, et al. Reliability, concurrent validity, and responsiveness of the Fugl-Meyer Assessment (FMA) for hemiplegic patients. Journal of Physical Therapy Science. 2012; 24(9):893-9. [DOI:10.1589/jpts.24.893]

[6] Barbosa NE, Forero SM, Galeano CP, Hernández ED, Landinez NS, Sunnerhagen KS, et al. Translation and cultural validation of clinical observational scales-the Fugl-Meyer assessment for post stroke sensorimotor function in Colombian Spanish. Disability and Rehabilitation. 2019; 41(19):2317-23. [PMID]

[7] Amano S, Umeji A, Uchita A, Hashimoto Y, Takebayashi T, Takahashi K, et al. Clinimetric properties of the Fugl-Meyer assessment with adapted guidelines for the assessment of arm function in hemiparetic patients after stroke. Topics in Stroke Rehabilitation. 2018; 25(7):500-8. [PMID]

[8] Hsueh IP, Hsu MJ, Sheu CF, Lee S, Hsieh CL, Lin JH. Psychometric comparisons of 2 versions of the Fugl-Meyer Motor Scale and 2 versions of the Stroke Rehabilitation Assessment of Movement. Neurorehabilitation and Neural Repair. 2008 22(6):737-44. [DOI:10.1177/1545968308315999] [PMID]

[9] Fugl-Meyer AR, Jääskö L, Leyman I, Olsson S, Steglind S. The post-stroke hemiplegic patient. 1. A method for evaluation of physical performance. Scandinavian Journal of Rehabilitation Medicine. 1975; 7(1):13-31. [PMID]

[10] Santisteban L, Térémetz M, Bleton JP, Baron JC, Maier MA Lindberg PG. Upper limb outcome measures used in stroke rehabilitation studies: A systematic literature review. Plos One. 2016; 11(5):e0154792. [PMID]

[11] Alt Murphy M, Resteghini C, Feys P, Lamers I. An overview of systematic reviews on upper extremity outcome measures after stroke. BMC Neurology. 2015; 15: 29. [DOI:10.1186/ s12883-015-0292-6] [PMID] [PMCID]

[12] Sullivan JE, Crowner BE, Kluding PM, Nichols D, Rose DK, Yoshida R, et al. Outcome measures for individuals with stroke: Process and recommendations from the American Physical Therapy Association neurology section task force. Physical Therapy. 2013; 93(10):1383-96. [DOI:10.2522/ ptj.20120492] [PMID]

[13] Duncan PW, Propst M, Nelson SG. Reliability of the FuglMeyer assessment of sensorimotor recovery following cerebrovascular accident. Physical Therapy. 1983; 63(10):1606-10. [DOI:10.1093/ptj/63.10.1606] [PMID] 
[14] Sanford J, Moreland J, Swanson LR, Stratford PW, Gowland C. Reliability of the Fugl-Meyer assessment for testing motor performance in patients following stroke. Physical Therapy. 1993; 73(7):447-54. [DOI:10.1093/ptj/73.7.447] [PMID]

[15] Page SJ, Levine P, Hade E. Psychometric properties and administration of the wrist/hand subscales of the Fugl-Meyer Assessment in minimally impaired upper extremity hemiparesis in stroke. Archives of Physical Medicine and Rehabilitation. 2012; 93(12):2373-6.e5. [DOI:10.1016/j.apmr.2012.06.017] [PMID] [PMCID]

[16] Lundquist CB, Maribo T. The Fugl-Meyer assessment of the upper extremity: Reliability, responsiveness and validity of the Danish version. Disability and Rehabilitation. 2017; 39(9):934-9. [PMID]

[17] Michaelsen SM, Rocha AS, Knabben RJ, Rodrigues LP, Fernandes CG. Translation, adaptation and inter-rater reliability of the administration manual for the Fugl-Meyer assessment. Revista Brasileira de Fisioterapia. 2011; 15(1):80-8 [DOI:10.1590/S1413-35552011000100013] [PMID]

[18] Platz T, Pinkowski C, van Wijck F, Kim IH, di Bella P, Johnson G. Reliability and validity of arm function assessment with standardized guidelines for the Fugl-Meyer Test, Action Research Arm Test and Box and Block Test: A multi centre study. Clinical Rehabilitation. 2005; 19(4):404-11. [DOI:10.1191/0269215505cr832oa] [PMID]

[19] Ezzati K, Salavati M, Abdollahi I, Shakeri H, Esmaili K. Persian translation, internal consistency and reliability of wolf motor function test. Caspian Journal of Neurological Sciences. 2017; 3(1):9-16. [DOI:10.18869/acadpub.cjns.3.8.9]

[20] Mokhtarinia HR, Hosseini A, Maleki-Ghahfarokhi A, Gabel CP, Zohrabi M. Cross-cultural adaptation, validity, and reliability of the Persian version of the spine functional index. Health and Quality of Life Outcomes. 2018; 16(1):95. [DOI:10.1186/s12955-018-0928-5] [PMID] [PMCID]

[21] Mokhtarinia HR, Shahbazi A, Abma FI, Gabel CP. Crosscultural adaptation, reliability, and validity of the work role functioning questionnaire 2.0 to Persian. Disability and Rehabilitation. 2021; 1-9. [PMID]

[22] Beaton DE, Bombardier C, Guillemin F, Ferraz MB Guidelines for the process of cross-cultural adaptation of self-report measures. Spine. 2000; 25(24):3186-91. [DOI:10.1097/00007632-200012150-00014] [PMID]

[23] Mokhtarinia H, Rafinia M, Shahbazi A, Khodaie Ardakani MR, Ghaedamini Harouni GR, Gabel CP. The cross-cultural adaptation of the smartphone addiction scale to Persian. Iranian Rehabilitation Journal. 2020; 18(1):91-8. [DOI:10.32598/ irj.18.1.919.1]

[24] Polit DF, Yang FM. Measurement and the measurement of change: A primer for the health professions. Philadelphia: Wolters Kluwer; 2016. https://www.google.com/books/ edition/Measurement_and_the_Measurement_of_Chang/ W2G80AEACAAJ?hl=en

[25] Mokhtarinia HR, Zareiyan A, Gabel CP. Cross-cultural adaptation, validity, and reliability of the Persian version of the Upper Limb Functional Index. Hand Therapy. 2021; 26(2):43-52. [DOI:10.1177/1758998320986832]

[26] Shrout PE, Fleiss JL. Intraclass correlations: Uses in assessing rater reliability. Psychological Bulletin. 1979; 86(2):420-8. [DOI:10.1037/0033-2909.86.2.420] [PMID]
[27] Koo TK, Li MY. A guideline of selecting and reporting intraclass correlation coefficients for reliability research. Journal of Chiropractic Medicine. 2016; 15(2):155-63. [DOI:10.1016/j. jcm.2016.02.012] [PMID] [PMCID]

[28] Fleiss JL, Levin B, Paik MC. Statistical methods for rates and proportions. New York: Wiley; 2013. https://www. google.com/books/edition/Statistical_Methods_for_Rates_ and_Propor/9VefO7a8GeAC?hl=en\&gbpv=0

[29] Terwee CB, Bot SD, de Boer MR, van der Windt DA, Knol DL, Dekker J, et al. Quality criteria were proposed for measurement properties of health status questionnaires. Journal of Clinical Epidemiology. 2007; 60(1):34-42. [DOI:10.1016/j. jclinepi.2006.03.012] [PMID]

[30] Terwee CB, Prinsen CAC, Chiarotto A, Westerman MJ, Patrick DL, Alonso J, et al. COSMIN methodology for evaluating the content validity of patient-reported outcome measures: A Delphi study. Quality of Life Research. 2018; 27(5):1159-70. [DOI:10.1007/s11136-018-1829-0] [PMID] [PMCID]

[31] Cecchi F, Carrabba C, Bertolucci F, Castagnoli C, Falsini C, Gnetti B, et al. Transcultural translation and validation of fugl-meyer assessment to Italian. Disability and Rehabilitation. 2020; 43(25):3717-22. [PMID]

[32] Busk H, Alt Murphy M, Korsman R, Skou ST, Wienecke T. Cross-cultural translation and adaptation of the Danish version of the Fugl-Meyer Assessment for post stroke sensorimotor function. Disability and Rehabilitation. 2021; 1-8. [PMID]

[33] Kim TL, Hwang SH, Lee WJ, Hwang JW, Cho I, Kim EH, et al. The Korean version of the Fugl-Meyer Assessment: Reliability and validity evaluation. Annals of Rehabilitation Medicine 2021; 45(2):83-98. [DOI:10.5535/arm.20225] [PMID] [PMCID]

[34] Page SJ, Hade E, Persch A. Psychometrics of the wrist stability and hand mobility subscales of the Fugl-Meyer Assessment in moderately impaired stroke. Physical Therapy. 2015; 95(1):103-8. [DOI:10.2522/pti.20130235] [PMID] [PMCID]

[35] Roman N, Miclaus R, Repanovici A, Nicolau C. Equal opportunities for stroke survivors' rehabilitation: A study on the validity of the upper extremity fugl-meyer assessment scale translated and adapted into Romanian. Medicina. 2020 56(8):409. [DOI:10.3390/medicina56080409] [PMID] [PMCID]

[36] Lin JH, Hsueh IP, Sheu CF, Hsieh CL. Psychometric properties of the sensory scale of the Fugl-Meyer Assessment in stroke patients. Clinical Rehabilitation. 2004; 18(4):391-7. [DOI:10.1191/0269215504cr737oa] [PMID] 